\title{
New Operational Matrix via Genocchi Polynomials for Solving Fredholm-Volterra Fractional Integro-Differential Equations
}

\author{
Jian Rong Loh, Chang Phang, and Abdulnasir Isah \\ Department of Mathematics and Statistics, Faculty of Science, Technology and Human Development, \\ Universiti Tun Hussein Onn Malaysia, Johor, Malaysia \\ Correspondence should be addressed to Chang Phang; pchang@uthm.edu.my
}

Received 14 September 2016; Revised 10 November 2016; Accepted 8 December 2016; Published 16 January 2017

Academic Editor: Luigi C. Berselli

Copyright (C) 2017 Jian Rong Loh et al. This is an open access article distributed under the Creative Commons Attribution License, which permits unrestricted use, distribution, and reproduction in any medium, provided the original work is properly cited.

It is known that Genocchi polynomials have some advantages over classical orthogonal polynomials in approximating function, such as lesser terms and smaller coefficients of individual terms. In this paper, we apply a new operational matrix via Genocchi polynomials to solve fractional integro-differential equations (FIDEs). We also derive the expressions for computing Genocchi coefficients of the integral kernel and for the integral of product of two Genocchi polynomials. Using the matrix approach, we further derive the operational matrix of fractional differentiation for Genocchi polynomial as well as the kernel matrix. We are able to solve the aforementioned class of FIDE for the unknown function $f(x)$. This is achieved by approximating the FIDE using Genocchi polynomials in matrix representation and using the collocation method at equally spaced points within interval $[0,1]$. This reduces the FIDE into a system of algebraic equations to be solved for the Genocchi coefficients of the solution $f(x)$. A few numerical examples of FIDE are solved using those expressions derived for Genocchi polynomial approximation. Numerical results show that the Genocchi polynomial approximation adopting the operational matrix of fractional derivative achieves good accuracy comparable to some existing methods. In certain cases, Genocchi polynomial provides better accuracy than the aforementioned methods.

\section{Introduction}

Fractional integro-differential equation (FIDE) is an equation that contains a fractional derivative term ${ }_{0} D_{x}^{\alpha} f(x)$ and an integral kernel operator term $\widetilde{K} f(x)=\int K(x, t, f(t)) d t$. An example is given as follows:

$$
\begin{aligned}
{ }_{0} D_{x}^{\alpha} f(x)= & h_{1}(x) f(x)+\int_{0}^{x} K_{1}(x, t) f(t) d t \\
& +\int_{0}^{1} K_{2}(x, t) f(t) d t+h_{2}(x) .
\end{aligned}
$$

Here, $f(x)$ is the unknown function to be solved and $K_{1}(x, t)$, $K_{2}(x, t)$ are the Volterra integral kernel and Fredholm integral kernel, respectively. ${ }_{0} D_{x}^{\alpha}$ is Caputo's fractional derivative and $h_{1}(x), h_{2}(x)$ are coefficient functions.

In the past few years, various wavelet operational matrices and polynomial operational matrices for both fractional differentiation and fractional integration have been derived one after another to solve various kinds of fractional differential equations (FDEs) and fractional integro-differential equations (FIDEs). Examples of polynomial operational matrices that have been proposed in the past are Shifted Legendre polynomials [1], shifted Chebyshev polynomials [2], and shifted Jacobi polynomials [3] to name a few. The operational matrix method is usually combined with methods such as collocation method, spectral tau method, and tau method to solve FDE and FIDE. In [4], Zhu and Fan have proposed Chebyshev wavelet operational matrix of fractional integration and applied it to solve a certain type of nonlinear fractional integro-differential equations (FIDEs). Apart from the operational matrix approach, there exist various approaches such as Adomian decomposition method [5], spectral tau method [6], Taylor expansion method [7], and Bernoulli matrix method [8]. In [9], Alshbool et al. have proposed a new Bernstein function to solve fractional order differential equations.

In this paper, different from the existing methods in numerically solving FIDE, we explore the application of a 
relatively new polynomial, namely, Genocchi polynomials, in the numerical solution of FIDE. Genocchi polynomial belongs to a larger family class of polynomials which is the Appell polynomial family. Throughout this paper, we denote Genocchi polynomials by $G_{n}(x)$. We apply these polynomials to solve the FIDEs given the advantage of Genocchi polynomials having smaller coefficients of each individual term and relatively lesser terms compared to classical orthogonal polynomials. Thus, this is expected to provide us with smaller computational errors.

The rest of the paper is organized as follows: Section 2 introduces preliminary definitions and properties of the Caputo fractional derivative. Section 3 gives basic definitions and properties of $G_{n}(x)$, function approximation by $G_{n}(x)$, and the analytical expression of $T_{(n, m)}=\int_{a}^{b} G_{n}(x) G_{m}(x) d x$ which is the integral of the product of two Genocchi polynomials. Section 4.1 derives the Genocchi polynomial operational matrix of Caputo's fractional derivative. Section 4.2 shows the way to approximate the integral kernel in terms of Genocchi polynomials and derives the analytical expression of the kernel matrix. Section 5 presents the general approach of approximating (1) into a system of algebraic equations and using collocation methods to solve (1). Section 6 describes the

general procedure of using Genocchi polynomials approximation to solve (1). Section 7 shows results of the proposed method. Section 8 sums up the findings of this paper.

\section{Preliminaries}

2.1. Fractional Calculus: Definitions. Fractional differentiation comes in different versions $[11,12]$. In this paper, we consider Caputo's fractional differentiation which provides a more realistic physical interpretation in real-life applications. Caputo's fractional derivative operator $D^{\alpha}$ of a function $f(t)$ is defined as follows:

Definition 1.

$$
\begin{aligned}
{ }_{0} D_{x}^{\alpha} f(x) & =\frac{1}{\Gamma(n-\alpha)} \int_{0}^{x}(x-t)^{n-\alpha-1} f^{(n)}(t) d t \\
n-1<\alpha \leq n, n \in \mathbb{N}, & \\
n & =\lceil\alpha\rceil .
\end{aligned}
$$

Below are some properties of Caputo's fractional derivatives:

$$
\begin{aligned}
{ }_{0} D_{x}^{\alpha} k & =0, \quad(k \text { is constant }) \\
{ }_{0} D_{x}^{\alpha} x^{\beta} & = \begin{cases}0, & \beta \in \mathbb{N} \cup\{0\}, \beta<\lceil\alpha\rceil \\
\frac{\Gamma(\beta+1)}{\Gamma(\beta+1-\alpha)} x^{\beta-\alpha}, & \beta \in \mathbb{N} \cup\{0\}, \beta \geq\lceil\alpha\rceil \text { or } \beta \notin \mathbb{N}, \beta>\lfloor\alpha\rfloor,\end{cases}
\end{aligned}
$$

where $\lceil\alpha\rceil$ denotes the smallest integer greater than or equal to $\alpha$ and $\lfloor\alpha\rfloor$ denotes the largest integer less than or equal to $\alpha$.

Caputo's fractional differential operator is linear:

$$
\begin{aligned}
& { }_{0} D_{x}^{\alpha}(\lambda f(x)+\mu h(x)) \\
& \quad=\lambda\left({ }_{0} D_{x}^{\alpha} f(x)\right)+\mu\left({ }_{0} D_{x}^{\alpha} h(x)\right),
\end{aligned}
$$

where $\lambda$ and $\mu$ are arbitrary constants.

\section{Genocchi Polynomials and Function Approximation}

3.1. Genocchi Polynomials: Definitions and Basic Properties. The Genocchi polynomials $G_{n}(x)$ are defined as follows [13$15]$.

Definition 2. The Genocchi polynomial $G_{n}(x)$ is defined by the generating function $Q(x, t)$ :

$$
\begin{aligned}
& Q(x, t)=\frac{2 t e^{x t}}{e^{t}+1}=\sum_{n=0}^{\infty} G_{n}(x) \frac{t^{n}}{n !}, \\
& G_{n}(x)=\sum_{k=0}^{n}\left(\begin{array}{l}
n \\
k
\end{array}\right) g_{n-k} x^{k}=2 B_{n}(x)-2^{n+1} B_{n}(x),
\end{aligned}
$$

where $g_{k}=2 B_{k}-2^{k+1} B_{k}$ is the Genocchi number. $B_{n}, B_{n}(x)$ are the Bernoulli number and Bernoulli polynomial, respectively.

Below are some of the important properties of Genocchi polynomials:

$$
\begin{aligned}
& \frac{d G_{n}(x)}{d x}=n G_{n-1}(x), \quad n \geq 1, \\
& \frac{d^{k} G_{n}(x)}{d x^{k}}= \begin{cases}0, & n \leq k \\
k !\left(\begin{array}{l}
n \\
k
\end{array}\right) G_{n-k}(x), & n>k\end{cases} \\
& k, n \in \mathbb{N} \cup\{0\},
\end{aligned}
$$$$
G_{n}(1)+G_{n}(0)=0, \quad n>1 \text {. }
$$

3.2. Function Approximation by Genocchi Polynomials. We may approximate a function $f(x)$ in terms of Genocchi polynomials $G_{n}(x)$ by the following infinite series:

$$
f(x)=\sum_{n=1}^{\infty} c_{n} G_{n}(x),
$$


where $G_{n}(x)$ are the Genocchi polynomials and the Genocchi coefficients $c_{n}$.

In practice, we truncate the infinite series up to $N$ number of Genocchi polynomials according to the desired accuracy of the problem into the following truncated Genocchi series:

$$
f(x) \simeq \sum_{n=1}^{N} c_{n} G_{n}(x),
$$

where in matrix notation,

$$
f(x)=\mathbf{C}^{T} \mathbf{G}(x),
$$

where $\mathbf{C}=\left[\begin{array}{llll}c_{1} & c_{2} & \cdots & c_{N}\end{array}\right]^{T}$ is the Genocchi coefficient vector. tor.

$\mathbf{G}(x)=\left[\begin{array}{llll}G_{1}(x) & G_{2}(x) & \cdots & G_{N}(x)\end{array}\right]^{T}$ is the Genocchi vec-

In this case, the Genocchi coefficients $c_{n}$ may be computed as follows:

$$
c_{n}=\frac{1}{2 n !}\left(f^{(n-1)}(0)+f^{(n-1)}(1)\right), \quad n=1,2, \ldots, N .
$$

3.3. Computing Genocchi Coefficients by Matrix Approach. Equation (10) fails to work for functions that are not $(n-1)$ differentiable at the points $x=0$ and $x=1$. An example is shown below.

Let $N=3 ; x^{3 / 2} \approx \sum_{n=1}^{3} c_{n} G_{n}(x)=c_{1} G_{1}(x)+c_{2} G_{2}(x)+$ $c_{3} G_{3}(x)$

$$
\begin{aligned}
c_{3} & =\frac{1}{2(3 !)}\left(\left.\frac{d^{2}}{d x^{2}} x^{3 / 2}\right|_{x=0}+\left.\frac{d^{2}}{d x^{2}} x^{3 / 2}\right|_{x=1}\right) \\
& =\frac{1}{2(3 !)}\left(\left.\frac{3}{4 \sqrt{x}}\right|_{x=0}+\left.\frac{3}{4 \sqrt{x}}\right|_{x=1}\right) .
\end{aligned}
$$

To avoid this problem that occurs in the case of differentiation, we may evaluate the Genocchi coefficients $c_{n}$ using Theorem 4. To prove Theorem 4, we first prove the following Theorem 3 which gives the analytical expression of the integral of the product of two Genocchi polynomials over an arbitrary interval $[a, b], 0 \leq a \leq b$ which will be used in the subsequent part of the paper.

Theorem 3. Given any two Genocchi polynomials $G_{n}(x)$, $G_{m}(x)$, for $x \geq 0$

$$
\begin{aligned}
\gamma_{n, m} & (x)=\int G_{n}(x) G_{m}(x) d x=\int_{0}^{x} G_{n}(x) G_{m}(x) d x \\
= & \sum_{r=0}^{n-1}(-1)^{r} \\
\cdot & \frac{n_{(r)}}{(m+1)^{(r+1)}}\left(G_{n-r}(x) G_{m+1+r}(x)-g_{n-r} g_{m+1+r}\right),
\end{aligned}
$$

where $g_{n-r}=G_{n-r}(0)$ is the Genocchi number, $n_{(r)},(m+1)^{(r+1)}$ are the falling and rising factorial, respectively. In particular, we have the following for $[a, b], 0 \leq a \leq b$

$$
\begin{aligned}
\gamma_{n, m}^{(a, b)} & =\int_{a}^{b} G_{n}(x) G_{m}(x) d x=\gamma_{n, m}(b)-\gamma_{n, m}(a) \\
& =\sum_{r=0}^{n-1}(-1)^{r} \frac{n_{(r)}}{(m+1)^{(r+1)}}\left(G_{n-r}(b) G_{m+1+r}(b)\right. \\
& \left.-G_{n-r}(a) G_{m+1+r}(a)\right), \\
\gamma_{n, m}^{(0,1)} & =\sum_{r=0}^{n-1}(-1)^{r} \frac{n_{(r)}}{(m+1)^{(r+1)}}\left(G_{n-r}(b) G_{m+1+r}(b)\right. \\
- & \left.g_{n-r} g_{m+1+r}\right) .
\end{aligned}
$$

Proof. Using the following expression:

$$
\begin{aligned}
\frac{d G_{m}(x)}{d x} & =m G_{m-1}(x), \\
\int_{a}^{b} G_{m}(x) d x & =\frac{G_{m+1}(b)-G_{m+1}(a)}{m+1}, \\
\int_{0}^{x} G_{m}(x) d x & =\frac{G_{m+1}(x)-g_{m+1}}{m+1} .
\end{aligned}
$$

We obtain

$$
\begin{aligned}
& \gamma_{n, m}(x)=\int G_{n}(x) G_{m}(x) d x, \\
& \gamma_{n, m}(x)=G_{n}(x)\left(\frac{G_{m+1}(x)-g_{m+1}}{m+1}\right) \\
& -\int\left(n G_{n-1}(x)\right) \frac{G_{m+1}(x)-g_{m+1}}{m+1} d x=G_{n}(x) \\
& \cdot\left(\frac{G_{m+1}(x)-g_{m+1}}{m+1}\right)-\frac{n}{m+1} \\
& \cdot \int G_{n-1}(x) G_{m+1}(x) d x+\frac{n}{m+1} \\
& \cdot g_{m+1} \int G_{n-1}(x) d x=G_{n}(x) \\
& \cdot\left(\frac{G_{m+1}(x)-g_{m+1}}{m+1}\right)-\frac{n}{m+1} \\
& \text {. } \int G_{n-1}(x) G_{m+1}(x) d x+\frac{1}{m+1} g_{m+1}\left(G_{n}(x)\right. \\
& \left.-g_{n}\right)=\left(\frac{G_{n}(x) G_{m+1}(x)-g_{n} g_{m+1}}{m+1}\right)-\frac{n}{m+1} \\
& \cdot \int G_{n-1}(x) G_{m+1}(x) d x \\
& \gamma_{n, m}(x)=\frac{G_{n}(x) G_{m+1}(x)-g_{n} g_{m+1}}{m+1}-\frac{n}{m+1}
\end{aligned}
$$




$$
\begin{aligned}
& \cdot \gamma_{n-1, m+1}(x)=\frac{G_{n}(x) G_{m+1}(x)-g_{n} g_{m+1}}{m+1} \\
& -\frac{n}{m+1}\left[\frac{G_{n-1}(x) G_{m+2}(x)-g_{n-1} g_{m+2}}{m+2}\right. \\
& \left.-\frac{n-1}{m+2} \gamma_{n-2, m+2}(x)\right] \\
& =\left(\frac{G_{n}(x) G_{m+1}(x)-g_{n} g_{m+1}}{m+1}\right)+(-1)^{1} \\
& \cdot \frac{n}{m+1}\left(\frac{G_{n-1}(x) G_{m+2}(x)-g_{n-1} g_{m+2}}{m+2}\right)+(-1)^{2} \\
& \cdot \frac{n(n-1)}{(m+1)(m+2)} \gamma_{n-2, m+2}(x) .
\end{aligned}
$$

Continuing this relation recursively for $n$ times, we arrive at

$$
\begin{aligned}
= & \sum_{r=0}^{n-1}(-1)^{r} \\
& \cdot \frac{n(n-1) \cdots(n-r+1)}{(m+1) \cdots(m+r)}\left(G_{n-r}(x) G_{m+1+r}(x)\right. \\
& \left.-g_{n-r} g_{m+1+r}\right)=\sum_{r=0}^{n-1}(-1)^{r} \\
& \cdot \frac{n_{(r)}}{(m+1)^{(r+1)}}\left(G_{n-r}(x) G_{m+1+r}(x)-g_{n-r} g_{m+1+r}\right) .
\end{aligned}
$$

Adopting the techniques used in [16] for function approximation, we prove the following theorem of function approximation using Genocchi polynomials.

Theorem 4. Let $f(x) \in L^{2}[0,1]$ and $\left\{G_{i}(x), i=1, \ldots, N\right\}$ be the set of Genocchi polynomials up to order $N$. Let $Y=$ $\operatorname{span}\left\{G_{1}(x), \ldots, G_{N}(x)\right\}$. Since $Y$ is a finite dimensional closed subspace of $L^{2}[0,1]$, then $\exists f^{*}(x) \in Y$ is the unique best approximation in Genocchi polynomials such that $f(x)$ can be approximated by unique coefficients $c_{n}$ :

$$
f(x) \approx f^{*}(x)=\sum_{n=1}^{N} c_{n} G_{n}(x)=\mathbf{C}^{T} \mathbf{G}(x) .
$$

The Genocchi coefficient matrix $\mathbf{C}$ consisting of the unique coefficients $c_{n}$ is given by the following:

$$
\mathbf{C}^{T}=\mathbf{F}^{T} \mathbf{T}^{(\mathbf{0 , 1})^{-1}}
$$

where $\mathbf{F}=\left[\int_{0}^{1} f(x) G_{m}(x) d x\right]_{N \times N}$ and $\mathbf{T}^{(\mathbf{0 , 1})}=$ $\left[\int_{0}^{1} G_{n}(x) G_{m}(x) d x\right]_{N \times N}$ as derived in Theorem 3 .
Proof.

$$
\begin{gathered}
f(x) \approx \sum_{n=1}^{N} c_{n} G_{n}(x), \\
\int_{0}^{1} f(x) G_{m}(x) d x=\sum_{n=1}^{N} c_{n} \int_{0}^{1} G_{n}(x) G_{m}(x) d x .
\end{gathered}
$$

Let $f_{m}=\int_{0}^{1} f(x) G_{m}(x) d x$. Then,

$$
\begin{aligned}
f_{m}=\sum_{n=1}^{N} c_{n} \int_{0}^{1} G_{n}(x) G_{m}(x) d x=\sum_{n=1}^{N} c_{n} \gamma_{n, m}^{(0,1)} & \\
& m=1, \ldots, N .
\end{aligned}
$$

Thus, we have a system of $N$ equations which is written in matrix representation as follows:

$$
\begin{gathered}
{\left[\begin{array}{c}
f_{1} \\
\vdots \\
f_{N}
\end{array}\right]=\left[c_{1}, \ldots, c_{N}\right]\left[\begin{array}{ccc}
\gamma_{1,1}^{(0,1)} & \cdots & \gamma_{1, N}^{(0,1)} \\
\gamma_{2,1}^{(0,1)} & \ldots & \gamma_{2, N}^{(0,1)} \\
\vdots & \ddots & \vdots \\
\gamma_{N, 1}^{(0,1)} & \cdots & \gamma_{N, N}^{(0,1)}
\end{array}\right],} \\
\mathbf{F}^{T}=\mathbf{C}^{T} \mathbf{T}^{(\mathbf{0 , 1})} .
\end{gathered}
$$

Therefore, the Genocchi coefficients matrix $\mathbf{C}$ is

$$
\mathbf{C}^{T}=\mathbf{F}^{T} \mathbf{T}^{(\mathbf{0 , 1})^{-1}}
$$

where $\gamma_{i, j}$ can be obtained from Theorem 3 .

\section{Main Result}

4.1. Genocchi Polynomial Operational Matrix of Caputo's Fractional Differentiation. In this section, we derive the analytical expression of the Genocchi polynomial operational matrix of Caputo's fractional derivative, which is the $N \times N$ matrix ${ }_{0} \mathbf{P}_{G}^{\alpha}$, where

$$
\begin{aligned}
{ }_{0} D_{x}^{\alpha} \mathbf{G}(x) & ={ }_{0} \mathbf{P}_{G}^{\alpha} \mathbf{G}(x), \\
{ }_{0} D_{x}^{\alpha}\left[\begin{array}{c}
G_{1} \\
G_{2} \\
\vdots \\
G_{N}
\end{array}\right] & =\left[\begin{array}{cccc}
\rho_{11} & \rho_{12} & \cdots & \rho_{1 N} \\
\rho_{21} & \rho_{22} & \cdots & \rho_{2 N} \\
\vdots & \cdots & \cdots & \vdots \\
\rho_{N 1} & \rho_{N 2} & \cdots & \rho_{N N}
\end{array}\right]\left[\begin{array}{c}
G_{1} \\
G_{2} \\
\vdots \\
G_{N}
\end{array}\right] .
\end{aligned}
$$

To derive ${ }_{0} \mathbf{P}_{G}^{\alpha}$, we first prove the following Lemmas 5 and 6 .

Lemma 5. Caputo's fractional derivative of fractional order $\alpha$ of a Genocchi polynomial of order $i$ is given by 
Advances in Mathematical Physics

5

$$
{ }_{0} D_{x}^{\alpha} G_{i}(x)= \begin{cases}\sum_{r=\lceil\alpha\rceil}^{i} \frac{i ! g_{i-r}}{(i-r) ! \Gamma(r-\alpha+1)} x^{r-\alpha}, & n-1<\alpha \leq n, n=\lceil\alpha\rceil \in \mathbb{N}, \quad i \geq \alpha \\ 0, & i<\alpha .\end{cases}
$$

Proof. For $n-1<\alpha \leq n, n=\lceil\alpha\rceil$

$$
\begin{aligned}
{ }_{0} D_{x}^{\alpha} G_{i}(x)=\frac{1}{\Gamma(n-\alpha)} \int_{0}^{x}(x-t)^{n-\alpha-1} \frac{d^{n}}{d t^{n}} G_{i}(t) d t \\
=\frac{1}{\Gamma(\lceil\alpha\rceil-\alpha)} \int_{0}^{x}(x-t)^{n-\alpha-1} \frac{d^{n}}{d t^{n}} \sum_{r=0}^{i}\left(\begin{array}{c}
i \\
r
\end{array}\right) g_{i-r} t^{r} d t \\
=\frac{1}{\Gamma(\lceil\alpha\rceil-\alpha)} \\
\cdot \sum_{r=0}^{i}\left(\begin{array}{l}
i \\
r
\end{array}\right) g_{i-r} \int_{0}^{x}(x-t)^{\lceil\alpha\rceil-\alpha-1} \frac{d^{\lceil\alpha\rceil}}{d t^{\lceil\alpha\rceil}} t^{r} d t \\
=\frac{1}{\Gamma(\lceil\alpha\rceil-\alpha)} \\
\quad \cdot \sum_{r=\lceil\alpha\rceil}^{i}\left(\begin{array}{l}
i \\
r
\end{array}\right) g_{i-r} \int_{0}^{x}(x-t)^{\lceil\alpha\rceil-\alpha-1}\lceil\alpha\rceil !\left(\begin{array}{c}
r \\
\lceil\alpha\rceil
\end{array}\right) t^{r-\lceil\alpha\rceil} d t \\
=\frac{1}{\Gamma(\lceil\alpha\rceil-\alpha)} \\
. \sum_{r=\lceil\alpha\rceil}^{i}\left(\begin{array}{l}
i \\
r
\end{array}\right)\lceil\alpha\rceil !\left(\begin{array}{c}
r \\
\lceil\alpha\rceil
\end{array}\right) g_{i-r} \int_{0}^{x}(x-t)^{\lceil\alpha\rceil-\alpha-1} t^{r-\lceil\alpha\rceil} d t,
\end{aligned}
$$

where $i \geq\lceil\alpha\rceil$. Substituting $t=x u$

$$
\begin{aligned}
& { }_{0} D_{x}^{\alpha} G_{i}(x)=\frac{1}{\Gamma(\lceil\alpha\rceil-\alpha)} \sum_{r=\lceil\alpha\rceil}^{i}\left(\begin{array}{c}
i \\
r
\end{array}\right)\lceil\alpha\rceil !\left(\begin{array}{c}
r \\
\lceil\alpha\rceil
\end{array}\right) g_{i-r} x^{r-\alpha} \\
& \cdot \int_{0}^{1}(1-u)^{\lceil\alpha\rceil-\alpha-1} u^{r-\lceil\alpha\rceil} d u=\frac{1}{\Gamma(\lceil\alpha\rceil-\alpha)} \\
& \cdot \sum_{r=\lceil\alpha\rceil}^{i}\left(\begin{array}{c}
i \\
r
\end{array}\right)\lceil\alpha\rceil !\left(\begin{array}{c}
r \\
\lceil\alpha\rceil
\end{array}\right) g_{i-r} x^{r-\alpha} B(r-\lceil\alpha\rceil+1,\lceil\alpha\rceil-\alpha) \\
& \quad=\sum_{r=\lceil\alpha\rceil}^{i} \frac{i ! g_{i-r}}{(i-r) ! \Gamma(r-\alpha+1)} x^{r-\alpha},
\end{aligned}
$$

where $B(x, y)=\int_{0}^{1} u^{x-1}(1-u)^{y-1} d u$ is the Beta function for $\operatorname{Re}(x), \operatorname{Re}(y)>0$.

Lemma 6. The matrix $\Theta_{N}$ defined as $\Theta_{N}=\int_{0}^{1}\left({ }_{0} D_{x}^{\alpha} \mathrm{G}(x)\right) *$ $\mathbf{G}^{T}(x) d x$ is given by the following:

$$
\Theta_{N}=\left[\theta_{i k}\right]_{N \times N}=\left[\int_{0}^{1}\left({ }_{0} D_{x}^{\alpha} G_{i}(x)\right) G_{k}(x) d x\right]_{N \times N},
$$

where

$$
\theta_{i k}=\sum_{r=\lceil\alpha\rceil}^{i} \sum_{p=0}^{k} \frac{i !\left(\begin{array}{c}
k \\
p
\end{array}\right) g_{i-r} g_{k-p}}{(i-r) !(r-\alpha+p+1) \Gamma(r-\alpha+1)} .
$$

Proof. From Lemma 5,

$$
\begin{aligned}
& { }_{0} D_{x}^{\alpha} G_{i}(x)=\sum_{r=\lceil\alpha\rceil}^{i} \frac{i ! g_{i-r}}{(i-r) ! \Gamma(r-\alpha+1)} x^{r-\alpha}, \\
& \left({ }_{0} D_{x}^{\alpha} G_{i}(x)\right) G_{k}(x) \\
& =\left(\sum_{r=\lceil\alpha\rceil}^{i} \frac{i ! g_{i-r}}{(i-r) ! \Gamma(r-\alpha+1)} x^{r-\alpha}\right) \\
& \cdot\left(\sum_{p=0}^{k}\left(\begin{array}{l}
k \\
p
\end{array}\right) g_{k-p} x^{p}\right) \\
& =\sum_{r=\lceil\alpha\rceil}^{i} \sum_{p=0}^{k} \frac{i ! g_{i-r}}{(i-r) ! \Gamma(r-\alpha+1)}\left(\begin{array}{l}
k \\
p
\end{array}\right) g_{k-p} x^{r-\alpha+p}, \\
& \int_{0}^{1}\left({ }_{0} D_{x}^{\alpha} G_{i}(x)\right) G_{k}(x) d x \\
& =\sum_{r=\lceil\alpha\rceil}^{i} \sum_{p=0}^{k} \frac{i ! g_{i-r}}{(i-r) ! \Gamma(r-\alpha+1)}\left(\begin{array}{l}
k \\
p
\end{array}\right) g_{k-p} \\
& \cdot \int_{0}^{1} x^{r-\alpha+p} d x \\
& =\sum_{r=\lceil\alpha\rceil}^{i} \sum_{p=0}^{k} \frac{i !\left(\begin{array}{c}
k \\
p
\end{array}\right) g_{i-r} g_{k-p}}{(i-r) !(r-\alpha+p+1) \Gamma(r-\alpha+1)} .
\end{aligned}
$$

Now, we can prove the following theorem that computes the operational matrix ${ }_{0} \mathbf{P}_{G}^{\alpha}$.

Theorem 7. Given a set of $G_{i}(x), i=1, \ldots, N$, of $N$ Genocchi polynomials, the Gnocchi polynomial operational matrix of Caput's fractional derivative of order $\alpha$ over the interval $[0,1]$ is the $N \times N$ matrix ${ }_{0} \mathbf{P}_{G}^{\alpha}$ and is given by

$$
{ }_{0} \mathbf{P}_{G}^{\alpha}=\Theta_{N}\left(\mathbf{T}^{(0,1)}\right)^{-1}
$$

where $\Theta_{N}$ is given in Lemma 6 and $\mathbf{T}^{(0,1)}=\left[\gamma_{n, m}^{(0,1)}\right]_{N \times N}$ with elements $\gamma_{n, m}^{(0,1)}$ given in Theorem 3 . 
Proof. From Lemma 5,

$$
\begin{aligned}
& { }_{0} D_{x}^{\alpha} \mathbf{G}(x)={ }_{0} \mathbf{P}_{G}^{\alpha} \mathbf{G}(x) \\
& \left({ }_{0} D_{x}^{\alpha} \mathbf{G}(x)\right) * \mathbf{G}^{T}(x)={ }_{0} \mathbf{P}_{G}^{\alpha} \mathbf{G}(x) \mathbf{G}^{T}(x), \\
& \int_{0}^{1}\left({ }_{0} D_{x}^{\alpha} \mathbf{G}(x)\right) \mathbf{G}^{T}(x) d x \\
& ={ }_{0} \mathbf{P}_{G}^{\alpha}\left(\int_{0}^{1} \mathbf{G}(x) \mathbf{G}^{T}(x) d x\right) \\
& \Theta_{N}={ }_{0} \mathbf{P}_{G}^{\alpha} \mathbf{T}^{(0,1)} \\
& { }_{0} \mathbf{P}_{G}^{\alpha}=\Theta_{N}\left(\mathbf{T}^{(0,1)}\right)^{-1} .
\end{aligned}
$$

Here, we justify the better accuracy of the new operational matrix of Caputo's fractional derivative due to Genocchi polynomials by comparing its errors and that obtained when using Shifted Legendre operational matrix. This may be due to the fact that Genocchi polynomials have some advantages over classical orthogonal polynomials in approximating function, such as lesser terms and smaller coefficients of individual terms. We define the absolute error and relative error respectively, as follows:

$$
\begin{aligned}
& \left|D^{\alpha} Y_{i}(x)-\left[\mathbf{P}^{\alpha} * \mathbf{Y}\right](i)\right|, \\
& \left|\frac{D^{\alpha} Y_{i}(x)-\left[\mathbf{P}^{\alpha} * \mathbf{Y}\right](i)}{D^{\alpha} Y_{i}(x)}\right|
\end{aligned}
$$

where $Y_{i}(x)$ is either the Genocchi polynomial $G_{i}(x)$ or Shifted Legendre polynomial $\widetilde{L}_{i}(x)$. In Figure 1, we compare the relative error obtained due to Genocchi polynomial operational matrix of fractional derivative (GPOMFD) and the Shifted Legendre operational matrix of fractional derivative (SLOMFD) derived in [17]. One clearly sees from the figure that the errors for GPOMFD become smaller than that of SLOMFD for $N \geq 3$ and their difference gets larger as $N$ increases over the interval $[0,1]$. Further, we also show the absolute error and relative error in Tables 1 and 2, respectively, for $\alpha=0.5$ at different points.

4.2. Approximation of Integral Kernel by Genocchi Polynomials. The integral kernel function $K(x, t)$ will be approximated by Genocchi polynomials for each variable $G_{i}(x), G_{j}(t)$ as follows:

$$
\begin{aligned}
K(x, t) & =\sum_{i=1}^{\infty} \sum_{j=1}^{\infty} k_{i j} G_{i}(x) G_{j}(t) \\
& \approx \sum_{i=1}^{N} \sum_{j=1}^{N} k_{i j} G_{i}(x) G_{j}(t)=\mathbf{G}^{T}(x) \mathbf{K}_{G} \mathbf{G}(t),
\end{aligned}
$$

where we refer to $\mathbf{K}_{G}$ as the kernel matrix by Genocchi polynomial. Theorem 8 provides the formula for obtaining the kernel matrix $\mathbf{K}_{G}$.

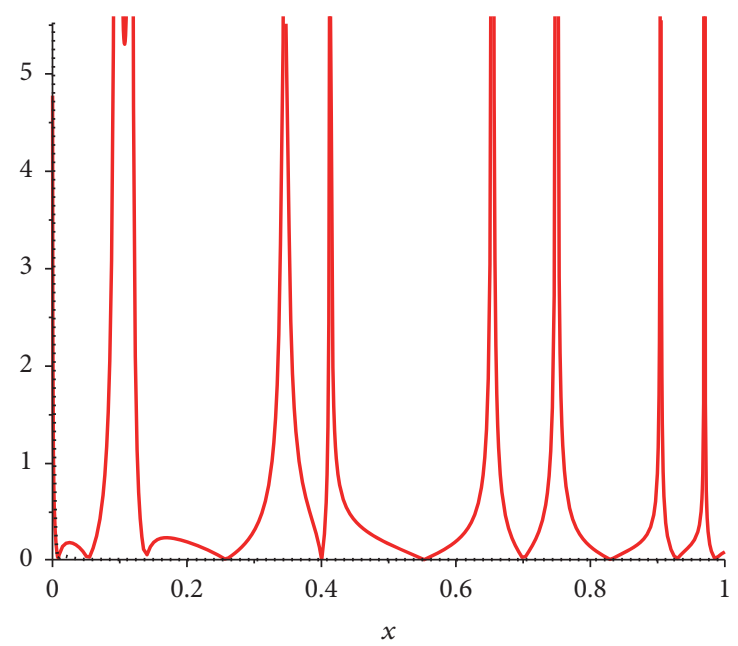

- $N=9$ Shifted Legendre polynomial OMFD relative error ..... $N=10$ Genocchi polynomial OMFD relative error

FIGURE 1: Comparison of relative error of operational matrix of fractional derivative (OMFD) with $\alpha=0.5$ between $N=9$ Shifted Legendre polynomials and $N=10$ Genocchi polynomials.

TABLE 1: Comparison of absolute error between SLOMFD and GPOMFD with $\alpha=0.5$ at points $x=0,0.5,1$.

\begin{tabular}{cccccccc}
\hline \multicolumn{4}{c}{ SLOMFD } & \multicolumn{3}{c}{ GPOMFD } \\
$N$ & $x=0$ & $x=0.5$ & $x=1$ & $N$ & $x=0$ & $x=0.5$ & $x=1$ \\
\hline 1 & 0.113121 & 0.001562 & 0.005656 & 2 & 0.113121 & 0.001306 & 0.005656 \\
3 & 0.696638 & 0.010167 & 0.036679 & 4 & 0.003583 & 0.000429 & 0.000549 \\
5 & 1.836044 & 0.030079 & 0.107732 & 6 & 0.008628 & 0.000272 & 0.001283 \\
7 & 3.773422 & 0.077279 & 0.273317 & 8 & 0.048392 & 0.004996 & 0.007218 \\
9 & 7.468363 & 0.257570 & 0.899849 & 10 & 0.440694 & 0.045400 & 0.065718 \\
\hline
\end{tabular}

Theorem 8. Let $K(x, t)$ be a two-variable continuous function in $C^{N-1}([0,1])$.

Then, $K(x, t)$ can be approximated in terms of Genocchi polynomials up to order $N$, that is, $K(x, t) \approx$ $\sum_{i=1}^{N} \sum_{j=1}^{N} k_{i j} G_{i}(x) G_{j}(t)=\mathbf{G}^{T}(x) \mathbf{K}_{G} \mathbf{G}(t), N \in \mathbb{N}$, where $\mathbf{G}$ is the Genocchi polynomial basis matrix and $\mathbf{K}_{G}$ is the $N \times N$ integral kernel matrix in Genocchi basis given by

$$
\mathbf{K}_{G}=\mathbf{T}^{(\mathbf{0 , 1})^{-1}} \mathbf{N}_{G} \mathbf{T}^{(\mathbf{0 , 1})^{-1}},
$$

where

$$
\begin{aligned}
& \mathbf{N}_{G}=\left[\eta_{p q}\right]_{N \times N} \\
& =\left[\int_{0}^{1} \int_{0}^{1} K(x, t) G_{p}(x) G_{q}(t) d x d t\right]_{N \times N}, \\
& \mathbf{T}^{(\mathbf{0 , 1})^{-1}}=\left[\gamma_{n, m}^{(0,1)}\right]_{N \times N}^{-1}=\left[\sum_{r=0}^{n-1}(-1)^{r}\right.
\end{aligned}
$$


TABLE 2: Comparison of relative error between SLOMFD and GPOMFD with $\alpha=0.5$ at points $x=0.1,0.6,1$.

\begin{tabular}{cccccccc}
\hline & & SLOMFD & & & \multicolumn{2}{c}{ GPOMFD } \\
$N$ & $x=0.1$ & $x=0.6$ & $x=1$ & $N$ & $x=0.1$ & $x=0.6$ & 0.0009516 \\
\hline 1 & 0.0061268 & 0.0009516 & 0.0025063 & 2 & 0.0061268 & 0.0025063 \\
3 & 0.0095486 & 0.0256030 & 0.0081265 & 4 & 0.0016094 & 0.0000698 & 0.0003038 \\
5 & 0.0235837 & 0.0171498 & 0.0159125 & 6 & 0.0014129 & 0.0000535 & 0.0002389 \\
7 & 0.1314007 & 0.5765935 & 0.0302776 & 8 & 0.0014150 & 0.0000529 & 0.0002372 \\
9 & 8.7021303 & 0.1871596 & 0.0797470 & 10 & 0.0014147 & 0.0000528 & 0.0002369 \\
\hline
\end{tabular}

$$
\begin{aligned}
& \cdot \frac{n_{(r)}}{(m+1)^{(r+1)}}\left(G_{n-r}(b) G_{m+1+r}(b)\right. \\
& \left.\left.-g_{n-r} g_{m+1+r}\right)\right]_{N \times N}^{-1} .
\end{aligned}
$$

Proof. From $K(x, t) \approx \mathbf{G}^{T}(x) \mathbf{K}_{G} \mathbf{G}(t)$,

$$
\begin{aligned}
& \mathbf{G}(x) K(x, t)=\mathbf{G}(x) \mathbf{G}^{T}(x) \mathbf{K}_{G} \mathbf{G}(t), \\
& \int_{0}^{1} \mathbf{G}(x) K(x, t) d x=\left(\int_{0}^{1} \mathbf{G}(x) \mathbf{G}^{T}(x) d x\right) \mathbf{K}_{G} \mathbf{G}(t) \\
& \quad=\mathbf{T}^{(0,1)} \mathbf{K}_{G} \mathbf{G}(t), \\
& \left(\int_{0}^{1} \mathbf{G}(x) K(x, t) d x\right) \mathbf{G}^{T}(t)=\mathbf{T}^{(0,1)} \mathbf{K}_{G} \mathbf{G}(t) \mathbf{G}^{T}(t), \\
& \int_{0}^{1} \int_{0}^{1} K(x, t) \mathbf{G}(x) \mathbf{G}^{T}(t) d x d t \\
& =\mathbf{T}^{(0,1)} \mathbf{K}_{G}\left(\int_{0}^{1} \mathbf{G}(t) \mathbf{G}^{T}(t) d t\right),
\end{aligned}
$$

where $\mathbf{T}^{(0,1)}=\int_{0}^{1} \mathbf{G}(x) \mathbf{G}^{T}(x) d x=\int_{0}^{1} \mathbf{G}(t) \mathbf{G}^{T}(t) d t$.

Define $\mathbf{N}_{G}=\int_{0}^{1} \int_{0}^{1} K(x, t) \mathbf{G}(x) \mathbf{G}^{T}(t) d x d t$. Thus,

$$
\begin{aligned}
& \mathbf{N}_{G}=\mathbf{T}^{(0,1)} \mathbf{K}_{G} \mathbf{T}^{(0,1)}, \\
& \mathbf{K}_{G}=\left(\mathbf{T}^{(0,1)}\right)^{-1} \mathbf{N}_{G}\left(\mathbf{T}^{(0,1)}\right)^{-1} .
\end{aligned}
$$

\section{Error Analysis}

Following the analysis made in [18], we derive the error estimates of numerical approximation using Genocchi polynomials considering the two following scenarios.

(1) If the exact solution $f(x)$ of (1) is known, and $f_{N}^{*}(x)$ is the numerical approximate solution using Genocchi polynomials up to order $N$, then we define the error estimation as follows:

$$
\delta_{N}=\left\|f(x)-f_{N}^{*}(x)\right\|_{2},
$$

where $\|y(x)\|_{2}=\int_{0}^{1} y^{2}(x) d x$. In the following theorem, we derive a bound for the $L_{2}$ error estimation of our Genocchi polynomial method.
Theorem 9. Let $f(x) \in L^{2}[0,1]$ be an arbitrary function. If $f(x)$ is approximated by truncated Genocchi polynomial series $f_{N}^{*}(x)=\sum_{n=1}^{N} c_{n} G_{n}(x)$ and $\left|f^{(n-1)}(x)\right|_{x \in[0,1]} \leq M$ (M is finite), then the error in $L_{2}$ is norm; $\delta_{N}=\left\|f(x)-f_{N}^{*}(x)\right\|_{2}$ for $N$ order approximation is bounded above by

$$
\delta_{N} \leq \sqrt{\sum_{n=N+1}^{\infty} \sum_{k=1}^{n} \frac{M^{2}}{2 *(n !)^{2}}\left(\begin{array}{l}
n \\
k
\end{array}\right)^{2} \frac{g_{n-k}^{2}}{2 k+1}} .
$$

Proof.

$$
\begin{aligned}
\delta_{N}^{2} & =\int_{0}^{1}\left|f(x)-f_{N}^{*}(x)\right|^{2} d x \\
& =\int_{0}^{1}\left|f(x)-\sum_{n=1}^{N} c_{n} G_{n}(x)\right|^{2} d x \\
& =\int_{0}^{1}\left|\sum_{n=N+1}^{\infty} c_{n} G_{n}(x)\right|^{2} d x \\
& \leq \int_{0}^{1} \sum_{n=N+1}^{\infty}\left|c_{n} G_{n}(x)\right|^{2} d x \\
& =\sum_{n=N+1}^{\infty} \int_{0}^{1}\left|c_{n}\right|^{2}\left|G_{n}(x)\right|^{2} d x, \\
\int_{0}^{1}\left|G_{n}(x)\right|^{2} d x & =\int_{0}^{1}\left|\sum_{k=1}^{n}\left(\begin{array}{l}
n \\
k
\end{array}\right) g_{n-k} x^{k}\right|^{2} d x \\
& \leq \sum_{k=1}^{n} \int_{0}^{1}\left(\begin{array}{l}
n \\
k
\end{array}\right)^{2} g_{n-k}^{2}\left|x^{k}\right|^{2} d x \\
& =\sum_{k=1}^{n}\left(\begin{array}{l}
n \\
k
\end{array}\right)^{2} g_{n-k}^{2} \int_{0}^{1} x^{2 k} d x \\
& =\sum_{k=1}^{n}\left(\begin{array}{l}
n \\
k
\end{array}\right)^{2} \frac{g_{n-k}^{2}}{2 k+1} .
\end{aligned}
$$

Therefore,

$$
\begin{aligned}
\delta_{N}^{2} & \leq \sum_{n=N+1}^{\infty}\left|c_{n}\right|^{2} \sum_{k=1}^{n}\left(\begin{array}{l}
n \\
k
\end{array}\right)^{2} \frac{g_{n-k}^{2}}{2 k+1} \\
& =\sum_{n=N+1}^{\infty}\left|\frac{1}{2 * n !}\left(f^{(n-1)}(0)+f^{(n-1)}(1)\right)\right|^{2} \sum_{k=1}^{n}\left(\begin{array}{l}
n \\
k
\end{array}\right)^{2}
\end{aligned}
$$




$$
\begin{gathered}
\cdot \frac{g_{n-k}^{2}}{2 k+1} \\
\leq \sum_{n=N+1}^{\infty} \sum_{k=1}^{n} \frac{1}{4 *(n !)^{2}}\left(\left|f^{(n-1)}(0)\right|^{2}+\left|f^{(n-1)}(1)\right|^{2}\right) \\
\cdot\left(\begin{array}{l}
n \\
k
\end{array}\right)^{2} \frac{g_{n-k}^{2}}{2 k+1}=\sum_{n=N+1}^{\infty} \sum_{k=1}^{n} \frac{1}{4 *(n !)^{2}}\left(2 M^{2}\right)\left(\begin{array}{l}
n \\
k
\end{array}\right)^{2} \\
\cdot \frac{g_{n-k}^{2}}{2 k+1}=\sum_{n=N+1}^{\infty} \sum_{k=1}^{n} \frac{M^{2}}{2 *(n !)^{2}}\left(\begin{array}{l}
n \\
k
\end{array}\right)^{2} \frac{g_{n-k}^{2}}{2 k+1}, \\
\delta_{N} \leq \sqrt{\sum_{n=N+1}^{\infty} \sum_{k=1}^{n} \frac{M^{2}}{2 *(n !)^{2}}\left(\begin{array}{l}
n \\
k
\end{array}\right)^{2} \frac{g_{n-k}^{2}}{2 k+1} .}
\end{gathered}
$$

Obviously, the estimation would become more precise if the order $N$ increases.

(2) In usual cases, the exact solution is unknown. So, first we obtain the approximate solution of order $N, f_{N}^{*}(x)$. Then, we obtain the approximate solution of order $N+1, f_{N}^{*}(x)$. Define the error estimation as follows:

$$
\epsilon_{N}=\left\|f_{N}^{*}(x)-f_{N+1}^{*}(x)\right\|_{2} .
$$

For a given desired error $\epsilon_{N}$, one is able to determine the smallest number $N$ which gives the desired error.

Theorem 10. Suppose $f_{N}^{*}(x)$ and $f_{N+1}^{*}(x)$ are numerical solutions for the unknown solution $f(x)$ of (1) with order $N, N+1$, respectively. The error estimation defined as $\epsilon_{N}=\| f_{N}^{*}(x)-$ $f_{N+1}^{*}(x) \|_{2}$ is convergent.

Proof.

$$
\begin{aligned}
\epsilon_{N} & =\left\|f_{N}^{*}(x)-f_{N+1}^{*}(x)\right\|_{2} \\
& =\left\|f_{N}^{*}(x)-f(x)+f(x)-f_{N+1}^{*}(x)\right\|_{2} \\
& \leq\left\|f_{N}^{*}(x)-f(x)\right\|_{2}+\left\|f(x)-f_{N+1}^{*}(x)\right\|_{2} \\
& =\delta_{N}+\delta_{N+1} .
\end{aligned}
$$

$\delta_{N}$ and $\delta_{N+1}$ are bounded by upper bound given in Theorem 9 and, in turn, are both convergent. Thus, $\epsilon_{N}$ is convergent too.

\section{Solving Fractional Integro-Differential Equation by Genocchi Polynomial Approximation}

As in any operational matrix method, we approximate each term in the following fractional integro-differential equation (FIDE) with Genocchi polynomials.

$$
\begin{aligned}
{ }_{0} D_{x}^{\alpha} f(x)= & h_{1}(x) f(x)+\int_{0}^{x} K_{1}(x, t) f(t) d t \\
& +\int_{0}^{1} K_{2}(x, t) f(t) d t+h_{2}(x), \\
f^{(i)}\left(x_{k}\right)= & y_{k}, \quad i, k=0, \ldots,\lceil\alpha\rceil-1 .
\end{aligned}
$$

For the FIDE in (1), we approximate each term using a set of Genocchi polynomials up to order $N$. The functions $h_{1}(x)$ and $h_{2}(x)$, which are known a priori in the FIDE, will not be approximated since this adds additional computational error to the numerical solution.

$$
\begin{aligned}
{ }_{0} D_{x}^{\alpha} f(x) & \approx \mathbf{C}_{0}^{T} \mathbf{P}_{G}^{\alpha} \mathbf{G}(x), \\
h(x) & \approx \mathbf{H}^{T} \mathbf{G}(x), \\
K_{1}(x, t) & \approx \mathbf{G}^{T}(x) \mathbf{K}_{\mathbf{1}} \mathbf{G}(t), \\
K_{2}(x, t) & \approx \mathbf{G}^{T}(x) \mathbf{K}_{\mathbf{2}} \mathbf{G}(t) .
\end{aligned}
$$

After approximation by Genocchi polynomial, the FIDE written in the matrix representation becomes the following:

$$
\begin{aligned}
\mathbf{G}^{T}(x)\left({ }_{0} \mathbf{P}_{G}^{\alpha}\right)^{T} \mathbf{C}= & h_{1}(x) \mathbf{G}^{T}(x) \mathbf{C} \\
& +\int_{0}^{x} \mathbf{G}^{T}(x) \mathbf{K}_{\mathbf{1}} \mathbf{G}(t) \mathbf{G}^{T}(t) \mathbf{C} d t \\
& +\int_{0}^{1} \mathbf{G}^{T}(x) \mathbf{K}_{\mathbf{2}} \mathbf{G}(t) \mathbf{G}^{T}(t) \mathbf{C} d t \\
& +h_{2}(x), \\
\mathbf{G}^{T}(x)\left({ }_{0} \mathbf{P}_{G}^{\alpha}\right)^{T} \mathbf{C}= & h_{1}(x) \mathbf{G}^{T}(x) \mathbf{C} \\
& +\mathbf{G}^{T}(x) \mathbf{K}_{\mathbf{1}} \mathbf{T}^{(0, x)} \mathbf{C} d t \\
& +\mathbf{G}^{T}(x) \mathbf{K}_{\mathbf{2}} \mathbf{T}^{(0,1)} \mathbf{C} d t \\
& +h_{2}(x),
\end{aligned}
$$

where $\mathbf{T}^{(0, x)}=\int_{0}^{x} \mathbf{G}(t) \mathbf{G}^{T}(t) d t$ and $\mathbf{T}^{(0,1)}=\int_{0}^{1} \mathbf{G}(t) \mathbf{G}^{T}(t) d t$.

Then, we select $N+1$ equally spaced points as the collocation points in the interval $[0,1]$; that is,

$$
v_{r}=\frac{r}{N}, \quad r=0, \ldots, N .
$$

After collocating (47), we obtain a system of $N+1$ algebraic equations consisting of $N$ Genocchi coefficients $\mathbf{C}=$ $\left[\begin{array}{lll}c_{1} & \cdots & c_{N}\end{array}\right]^{T}$. Given $\lceil\alpha\rceil$ initial conditions, $f^{(i)}\left(x_{k}\right)=y_{k}$, $i, k=0, \ldots,\lceil\alpha\rceil-1$, we select $N-\lceil\alpha\rceil$ equations out of the $N$ algebraic equations and solve $\mathbf{C}$ numerically. Thus, we obtain the approximate solution $f_{N}^{*}(x)=\mathbf{C}^{T} \mathbf{G}(x)$ of order $N$.

\section{Numerical Examples}

We present some examples of FIDEs which are solved using the proposed method of Genocchi polynomial approximation and Genocchi polynomial operational matrix of fractional derivative (GPOMFD). As in [8], we compute the maximum error function of the approximate function $f_{N}^{*}(x)$ of order $N$ for each numerical solution obtained as follows:

$$
\begin{aligned}
e_{\infty}(N) & =\left\|f(x)-f_{N}^{*}(x)\right\|_{\infty} \\
& =\max \left|f(x)-f_{N}^{*}(x)\right|, \quad a \leq x \leq b,
\end{aligned}
$$




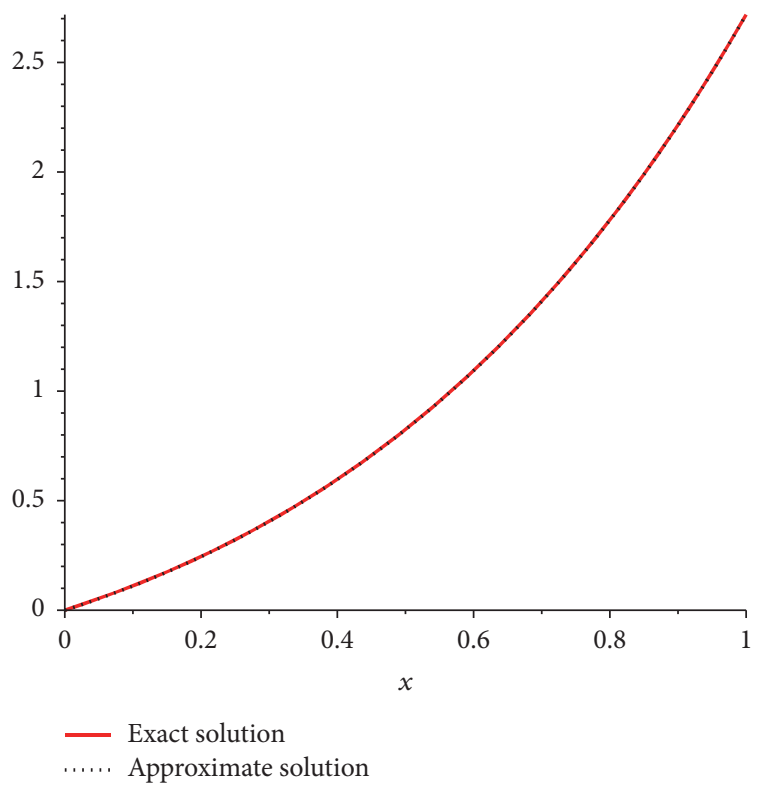

FIGURE 2: Comparison of exact solution $f(x)$ and approximate solution $f^{*}(x)$ for Example 2.

where $f(x)$ is the exact solution and $f_{N}^{*}(x)$ is the approximate solution of order $N$. Also, we compute the absolute error of $N$ order approximation at a particular point $x \in[0,1]$ as follows:

$$
e_{N}(x)=\left\|f(x)-f_{N}^{*}(x)\right\| \text {. }
$$

Example 1 (integer case). Given

$$
{ }_{0} D_{x}^{2} f(x)=-x^{2} f(x)+\int_{0}^{x} x t f(t) d t+h_{2}(x)
$$

with initial conditions $f(0)=2, f^{(1)}(0)=0 . h_{2}(x)=$ $-(1 / 30) x^{7}+(1 / 5) x^{6}+(1 / 4) x^{5}-x^{4}+x^{3}+(2 / 5) x^{2}-2$. The exact solution is $f(x)=(1 / 5) x^{4}-x^{2}-2$. Using $N=5$, that is, $f_{5}^{*}(x)=\sum_{k=1}^{5} c_{i} G_{i}(x)$, the Genocchi polynomial method gives us the exact solution $f_{5}^{*}(x)=(1 / 5) x^{4}-x^{2}-2$.

Example 2 (integer case). As for Example 3 in [8], given

$$
{ }_{0} D_{x}^{1} f(x)=x e^{x}-e^{x}-x+\int_{0}^{1} x f(t) d t
$$

with initial condition $f(0)=0$, the exact solution is $f(x)=$ $x e^{x}$. Figure 2 shows both $f(x)$ and $f_{11}^{*}(x)$ over the interval $[0,1]$. Tables 3 and 4 show the numerical results of the exact solutions and approximate solutions $\left(f_{11}^{*}(x), f_{17}^{*}(x)\right)$ by Genocchi polynomial for $N=11,17$ and approximate solutions $\left(y_{10}(x), y_{16}(x)\right)$ by Bernoulli matrix [8] together with absolute errors for both methods. For comparison, Genocchi polynomial approximation of $N+1$ (i.e., degree $N$ ) is compared with the Bernoulli matrix method of order $N$. Therefore, the Genocchi method of order 11 (i.e., degree 10 ) is compared with the Bernoulli method of $N=10$.
Example 3 (integer case). We consider the following problem as in [8]:

$$
{ }_{0} D_{x}^{1} f(x)=f(x)+\int_{0}^{1} e^{x t} f(t) d t+\frac{1-e^{x+1}}{x+1}
$$

with the initial condition $f(0)=1$. The exact solution is $f(x)=e^{x}$. Table 5 shows the $L_{\infty}$ error of the Genocchi approximate solutions for order $N=7, \ldots, 10$ and that of Bernoulli method [8] for the corresponding order of $N=$ $7, \ldots, 9$.

Example 4 (fractional case). We consider the following problem in [10].

$$
\begin{aligned}
{ }_{0} D_{x}^{1.7} f(x)= & \int_{0}^{x}(x-t) f(t) d t+\int_{0}^{1}(x+t) f(t) d t \\
& +g(x)
\end{aligned}
$$

with the initial conditions $f^{(1)}(0)=f(0)=0$ and $g(x)=$ $(\Gamma(3) / \Gamma(1.3)) x^{0.3}+(\Gamma(4) / \Gamma(2.3)) x^{1.3}-x^{4} / 12-x^{5} / 20-7 x / 12-$ $9 / 20$. The exact solution is $f(x)=x^{2}+x^{3}$. Table 6 shows the numerical results of the Genocchi approximate solutions, $f_{8}^{*}(x)$ for $N=8$, and Legendre wavelet method (LWM) approximate solution for $\operatorname{LWM}_{k=4, M=2}(x)$ [10]. Though the exact solution is a polynomial, one is unable to obtain the exact solution. This is because the operational matrix does not give exact approximation of fractional derivative ${ }_{0} D_{x}^{1.7} f(x)$, that is,

$$
\left\|D_{0}^{1.7} f(x)-\mathbf{C}_{0}^{T} \mathbf{P}_{G}^{1.7} \mathbf{G}(x)\right\|_{L^{2}} \neq 0 .
$$

Example 5 (fractional case). We consider the following problem in [10].

$$
\begin{aligned}
{ }_{0} D_{x}^{2.3} f(x)= & \frac{1}{4} \int_{0}^{x}(x-t) f(t) d t+\frac{1}{2} \int_{0}^{1} x t f(t) d t \\
& +g(x)
\end{aligned}
$$

with the initial conditions $f^{(2)}(0)=f^{(1)}(0)=f(0)=0$ and $g(x)=(\Gamma(4.5) / \Gamma(2.2)) x^{1.2}-x^{5.5} / 99-x / 11$. The exact solution is $f(x)=x^{7 / 2}$. Table 7 shows the numerical results of the Genocchi approximate solutions $f_{8}^{*}(x)$ for $N=8$ and $f_{9}^{*}(x)$ for $N=9$ and Legendre wavelet method (LWM) approximate solution $\left(\mathrm{LWM}_{k=3, M=2}(x)\right)$ in [10]. We manage to obtain comparable errors for Genocchi polynomials with that of Legendre wavelets which are known to be a more powerful tool with a higher accuracy than its counterpart, Legendre polynomials.

Example 6 (fractional case and solution is unknown). We consider the following problem:

$$
\begin{aligned}
{ }_{0} D_{x}^{1.5} f(x)= & \frac{1}{4} \int_{0}^{x}(x-t) f(t) d t+\frac{1}{2} \int_{0}^{1} x t f(t) d t \\
& +g(x)
\end{aligned}
$$


TABLE 3: Comparison of absolute errors for Example 2 using $N=11$ orders of Genocchi polynomials and Bernoulli method $N=10$ [8].

\begin{tabular}{lcccc}
\hline$x$ & Exact $f(x)$ & Genocchi $f_{11}^{*}(x)$ & Genocchi $e_{11}(x)$ & Bernoulli $e_{10}(x)[8]$ \\
\hline 0.0 & 0.0000000000000000 & 0.0000000000000000 & $0.00000 E+00$ & $6.33107 E-16$ \\
0.1 & 0.1105170918075647 & 0.1105170918090843 & $1.51950 E-12$ & $1.50312 E-10$ \\
0.2 & 0.2442805516320338 & 0.2442805516333059 & $1.27210 E-12$ & $6.01255 E-10$ \\
0.3 & 0.4049576422728009 & 0.4049576422741780 & $1.37710 E-12$ & $1.35331 E-09$ \\
0.4 & 0.5967298790565080 & 0.5967298790578598 & $1.35200 E-12$ & $2.41699 E-09$ \\
0.5 & 0.8243606353500640 & 0.8243606353514914 & $1.42950 E-12$ & $3.89875 E-09$ \\
0.6 & 1.0932712802343040 & 1.0932712802357070 & $1.40858 E-12$ & $6.46841 E-09$ \\
0.7 & 1.4096268952293330 & 1.4096268952309410 & $1.61360 E-12$ & $1.31824 E-08$ \\
0.8 & 1.7804327427939730 & 1.7804327427938810 & $8.72000 E-14$ & $3.51353 E-08$ \\
0.9 & 2.2136428000412540 & 2.2136427999488390 & $9.24140 E-11$ & $1.06298 E-07$ \\
1.0 & 2.7182818284590450 & 2.7182818284628360 & $3.79650 E-12$ & $3.17917 E-07$ \\
\hline
\end{tabular}

TABLE 4: Comparison of absolute errors for Example 2 using $N=17$ orders of Genocchi polynomials and Bernoulli method $N=16$ [8].

\begin{tabular}{lcccc}
\hline$x$ & Exact $f(x)$ & Genocchi $f_{17}^{*}(x)$ & Genocchi $e_{17}(x)$ & Bernoulli $e_{16}(x)[8]$ \\
\hline 0.0 & 0.0000000000000000 & 0.0000000000000000 & $5.80000 E-17$ & $3.00738 E-15$ \\
0.1 & 0.1105170918075647 & 0.1105170918075627 & $2.00000 E-15$ & $2.34124 E-15$ \\
0.2 & 0.2442805516320338 & 0.2442805516320308 & $3.00000 E-15$ & $1.23102 E-15$ \\
0.3 & 0.4049576422728009 & 0.4049576422727964 & $4.50000 E-15$ & $4.89821 E-16$ \\
0.4 & 0.5967298790565080 & 0.5967298790565028 & $5.20000 E-15$ & $2.87680 E-15$ \\
0.5 & 0.8243606353500640 & 0.8243606353500572 & $6.80000 E-15$ & $5.76338 E-15$ \\
0.6 & 1.0932712802343040 & 1.0932712802342920 & $1.20000 E-14$ & $8.76098 E-15$ \\
0.7 & 1.4096268952293330 & 1.4096268952293210 & $1.20000 E-14$ & $1.25357 E-14$ \\
0.8 & 1.7804327427939730 & 1.7804327427939550 & $1.80000 E-14$ & $1.65325 E-14$ \\
0.9 & 2.2136428000412540 & 2.2136428000412350 & $1.90000 E-14$ & $2.76347 E-14$ \\
1.0 & 2.7182818284590450 & 2.7182818284590240 & $2.10000 E-14$ & $7.29318 E-14$ \\
\hline
\end{tabular}

TABLE 5: Comparison of $L_{\infty}$ error for Example 3 using $N=7,8,9$ orders of Genocchi polynomials and that of Bernoulli method [8].

\begin{tabular}{lccc}
\hline$N$ & Genocchi $e_{\infty}(N)$ & $N$ & Bernoulli $e_{\infty}(N)[8]$ \\
\hline 7 & $2.685084410123100 E-07$ & - & - \\
8 & $4.733793870463971 E-08$ & 7 & $1.11973 E-04$ \\
9 & $8.708190200000000 E-08$ & 8 & $1.14161 E-05$ \\
\hline
\end{tabular}

with the initial conditions $f^{(1)}(0)=f(0)=0$ and $g(x)=$ $(1 / 60) x^{6}-(1 / 48) x^{4}+(1 / 24) x^{3}-(3 / 8) x^{2}-(128 / 5 \sqrt{\pi}) x^{5 / 2}-$ $(13 / 24) x+4(\sqrt{x} / \sqrt{\pi})$. The solution is unknown. Using Theorem 10 which follows the concept given in [18], we calculate $\epsilon_{N}=\left\|f_{N}^{*}(x)-f_{N+1}^{*}(x)\right\|_{2}=\sqrt{\int_{0}^{1}\left|f_{N}^{*}(x)-f_{N+1}^{*}(x)\right|^{2} d x}$ for $N=4,5,6,7,8$ shown in Table 8. Table 9 shows $\Delta_{N}=$ $\left|f_{N}^{*}(x)-f_{N+1}^{*}(x)\right|$, the so-called $N$ th level error estimate [18].

Based on the results of Table 8 which is calculated using Theorem 10, one should choose $N \geq 5$ if $\epsilon_{N}<0.005$ is to be achieved, where 0.005 is called the tolerance.

\section{Conclusion}

In this paper, FIDE is solved with a numerical approximation scheme using Genocchi polynomial and Genocchi polynomial operational matrix of fractional derivative (GPOMFD). To overcome the problem that occurs for the classical formula of Genocchi coefficients in the case of nondifferentiable function, we use the matrix approach to determine the Genocchi coefficients of an arbitrary integrable function. Also, we derive the analytical expression of $\mathbf{T}$ which is the integral of the product of Genocchi polynomials, $\mathbf{K}_{G}$ which is the integral kernel matrix, and ${ }_{0} \mathbf{P}_{G}^{\alpha}$ which is the Genocchi polynomial operational matrix of Caputo's fractional derivatives. The approximation of Genocchi polynomial operational matrix of fractional derivative (GPOMFD) is shown to achieve smaller error than that of Shifted Legendre polynomial operational matrix of fractional derivative (SLOMFD). This is due to the fact that Genocchi polynomials have smaller coefficients for each $x^{n}$-term than the Shifted Legendre polynomial. Applying Genocchi polynomial approximation, the FIDE is transformed into a system of algebraic equations of Genocchi coefficients $\mathbf{C}$ of the unknown function $f(x)$. Using collocation method of equally spaced points over the interval $[0,1]$ together with the initial conditions given, we are able to solve FIDE with good accuracy. Comparison with the Bernoulli matrix method [8] and the Legendre wavelet method [10] indicates that Genocchi polynomial can achieve comparable results as those of the aforementioned methods which are known to have better accuracy than Legendre polynomial. Estimation errors occur when one tries to solve the FIDE in the case when the derivative ${ }_{0} D_{x}^{\alpha}$ is of fractional order. 
TABLE 6: Comparison of approximate solutions for Example 4 using $N=8$ order Genocchi polynomials and Legendre wavelet method (LWM) [10] for $k=4$ and $M=2$.

\begin{tabular}{lccccc}
\hline$x$ & Exact & Genocchi & Genocchi & LWM & LWM \\
& $f(x)$ & $f_{8}^{*}(x)$ & $e_{8}(x)$ & $f_{k=4, M=2}^{*}(x)$ & 0.000024 \\
0.000003 & 0.015382 & $3.50000 E-06$ & 0.015822 & $2.40000 E-05$ \\
0.125 & 0.000000 & 0.074814 & $3.31010 E-03$ & 0.075531 & $1.75600 E-03$ \\
0.250 & 0.017578 & 0.189006 & $4.35300 E-03$ & 0.192880 & $2.59400 E-03$ \\
0.375 & 0.078125 & 0.364240 & $1.07600 E-02$ & 0.361498 & $1.79000 E-04$ \\
0.500 & 0.193359 & 0.608990 & $2.57750 E-02$ & 0.622950 & $1.18150 E-02$ \\
0.625 & 0.375000 & 0.936805 & $4.75700 E-02$ & 0.930901 & $5.34740 E-02$ \\
0.750 & 0.634765 & 1.365460 & $7.00800 E-02$ & 1.391340 & $4.42000 E-02$ \\
0.875 & 0.984375 & & & 02 \\
\hline
\end{tabular}

TABLE 7: Comparison of absolute errors for Example 5 using $N=$ 8 order of Genocchi polynomials with respect to Legendre wavelet method (LWM $k=3, M=2$ ) of [10].

\begin{tabular}{lccc}
\hline$x$ & Genocchi $e_{8}(x)$ & Genocchi $e_{9}(x)$ & LWM $e_{k=3, M=2}(x)[10]$ \\
\hline 0.000 & $1.10000 E-25$ & $1.20000 E-25$ & 0 \\
0.125 & $1.60950 E-04$ & $1.54175 E-04$ & $2.2475 E-04$ \\
0.250 & $6.47020 E-04$ & $6.30863 E-04$ & $5.6882 E-03$ \\
0.375 & $1.26848 E-03$ & $1.27497 E-03$ & $8.0634 E-03$ \\
0.500 & $1.94127 E-03$ & $2.01243 E-03$ & $2.3712 E-04$ \\
0.625 & $2.66168 E-03$ & $2.82528 E-03$ & $2.8084 E-02$ \\
0.750 & $3.43505 E-03$ & $3.70353 E-03$ & $3.1612 E-02$ \\
0.875 & $4.25649 E-03$ & $4.63810 E-03$ & $3.3599 E-02$ \\
\hline
\end{tabular}

TABLE 8: $\epsilon_{N}=\sqrt{\int_{0}^{1}\left|f_{N}^{*}(x)-f_{N+1}^{*}(x)\right|^{2} d x}$ of Example 6 for $N=$ $4,5,6,7,8$ of Genocchi polynomials.

\begin{tabular}{lcccc}
\hline$\epsilon_{4}$ & $\epsilon_{5}$ & $\epsilon_{6}$ & $\epsilon_{7}$ & $\epsilon_{8}$ \\
\hline 0.295460 & 0.000466 & 0.000461 & 0.000337 & 0.000318 \\
\hline
\end{tabular}

TABLE 9: $\Delta_{N}=\left|f_{N}^{*}(x)-f_{N+1}^{*}(x)\right|$ of Example 6 for $N=4,5,6,7,8$ of Genocchi polynomials.

\begin{tabular}{lccccc}
\hline$x$ & $\Delta_{4}$ & $\Delta_{5}$ & $\Delta_{6}$ & $\Delta_{7}$ & $\Delta_{8}$ \\
\hline 0.00 & 0.000000 & 0.000000 & 0.000000 & 0.000000 & 0.000000 \\
0.10 & 0.027424 & 0.000156 & 0.000099 & 0.000047 & 0.000004 \\
0.20 & 0.092740 & 0.000364 & 0.000091 & 0.000073 & 0.000142 \\
0.30 & 0.174125 & 0.000393 & 0.000120 & 0.000267 & 0.000237 \\
0.40 & 0.254577 & 0.000185 & 0.000379 & 0.000340 & 0.000223 \\
0.50 & 0.321910 & 0.000189 & 0.000507 & 0.000290 & 0.000243 \\
0.60 & 0.368759 & 0.000577 & 0.000435 & 0.000276 & 0.000349 \\
0.70 & 0.392579 & 0.000786 & 0.000268 & 0.000416 & 0.000406 \\
0.80 & 0.395643 & 0.000630 & 0.000251 & 0.000605 & 0.000322 \\
0.90 & 0.385042 & 0.000020 & 0.000653 & 0.000503 & 0.000363 \\
1.00 & 0.372688 & 0.001202 & 0.001572 & 0.000118 & 0.000917 \\
\hline
\end{tabular}

\section{Competing Interests}

The authors declare that there is no conflict of interests regarding the publication of this manuscript.

\section{Acknowledgments}

This work was supported in part by Malaysia UTHM FRGS Grant (Vot 1433).

\section{References}

[1] M. H. Akrami, M. H. Atabakzadeh, and G. H. Erjaee, "The operational matrix of fractional integration for shifted Legendre polynomials," Iranian Journal of Science and Technology, vol. 37, no. 4, pp. 439-444, 2013.

[2] A. H. Bhrawy and A. S. Alofi, "The operational matrix of fractional integration for shifted Chebyshev polynomials," Applied Mathematics Letters. An International Journal of Rapid Publication, vol. 26, no. 1, pp. 25-31, 2013.

[3] A. H. Bhrawy, M. M. Tharwat, and M. A. Alghamdi, "A new operational matrix of fractional integration for shifted Jacobi polynomials," Bulletin of the Malaysian Mathematical Sciences Society, Series 2, vol. 37, no. 4, pp. 983-995, 2014.

[4] L. Zhu and Q. Fan, "Solving fractional nonlinear Fredholm integro-differential equations by the second kind Chebyshev wavelet," Communications in Nonlinear Science and Numerical Simulation, vol. 17, no. 6, pp. 2333-2341, 2012.

[5] S. S. Ray and R. K. Bera, "Analytical solution of the Bagley Torvik equation by Adomian decomposition method," Applied Mathematics and Computation, vol. 168, no. 1, pp. 398-410, 2005.

[6] P. Mokhtary and F. Ghoreishi, "The $\mathrm{L}^{2}$-convergence of the Legendre spectral Tau matrix formulation for nonlinear fractional integro differential equations," Numerical Algorithms, vol. 58, no. 4, pp. 475-496, 2011.

[7] L. Huang, X.-F. Li, Y. Zhao, and X.-Y. Duan, "Approximate solution of fractional integro-differential equations by Taylor expansion method," Computers \& Mathematics with Applications, vol. 62, no. 3, pp. 1127-1134, 2011.

[8] A. H. Bhrawy, E. Tohidi, and F. Soleymani, "A new Bernoulli matrix method for solving high-order linear and nonlinear Fredholm integro-differential equations with piecewise intervals," Applied Mathematics and Computation, vol. 219, no. 2, pp. 482-497, 2012.

[9] M. H. T. Alshbool, A. S. Bataineh, I. Hashim, and O. R. Isik, "Solution of fractional-order differential equations based on the operational matrices of new fractional Bernstein functions," Journal of King Saud University Science, vol. 28, pp. 119-202, 2015.

[10] Z. Meng, L. Wang, H. Li, and W. Zhang, "Legendre wavelets method for solving fractional integro-differential equations," 
International Journal of Computer Mathematics, vol. 92, no. 6, pp. 1275-1291, 2015.

[11] I. Podlubny, Fractional Differential Equations : An Introduction to Fractional Derivatives, Fractional Differential Equations, vol. 198 of Some Methods of Their Solution and Some of Their Applications, Academic Press, 1998.

[12] A. A. Kilbas, H. M. Srivastava, and J. J. Trujillo, Theory and applications of fractional differential equations, vol. 204 of NorthHolland Mathematics Studies, Elsevier Science, Amsterdam, The Netherlands, 1st edition, 2006.

[13] S. Araci, "Novel identities for q-genocchi numbers and polynomials," Journal of Function Spaces and Applications, vol. 2012, Article ID 214961, 13 pages, 2012.

[14] S. Araci, "Novel identities involving Genocchi numbers and polynomials arising from applications of umbral calculus," Applied Mathematics and Computation, vol. 233, pp. 599-607, 2014.

[15] A. F. Horadam, "Genocchi polynomials", in Proceedings of the 4th International Conference on Fibonacci Numbers and Their Applications, pp. 145-166, Kluwer Academic, Winston-Salem, NC, USA, 1991.

[16] E. Keshavarz, Y. Ordokhani, and M. Razzaghi, "A numerical solution for fractional optimal control problems via Bernoulli polynomials," Journal of Vibration and Control, vol. 22, no. 18, 2015.

[17] A. Saadatmandi and M. Dehghan, "A new operational matrix for solving fractional-order differential equations," Computers \& Mathematics with Applications, vol. 59, no. 3, pp. 1326-1336, 2010.

[18] Y. Chen, X. Ke, and Y. Wei, "Numerical algorithm to solve system of nonlinear fractional differential equations based on wavelets method and the error analysis," Applied Mathematics and Computation, vol. 251, pp. 475-488, 2015. 


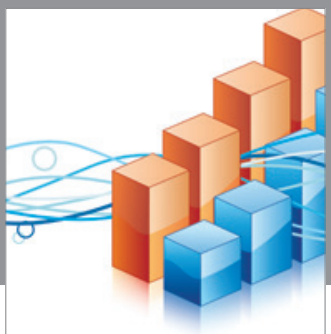

Advances in

Operations Research

vatem alat4

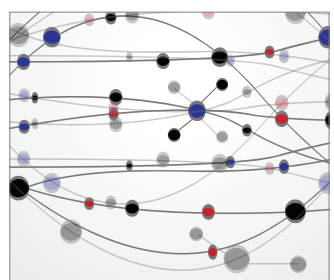

\section{The Scientific} World Journal
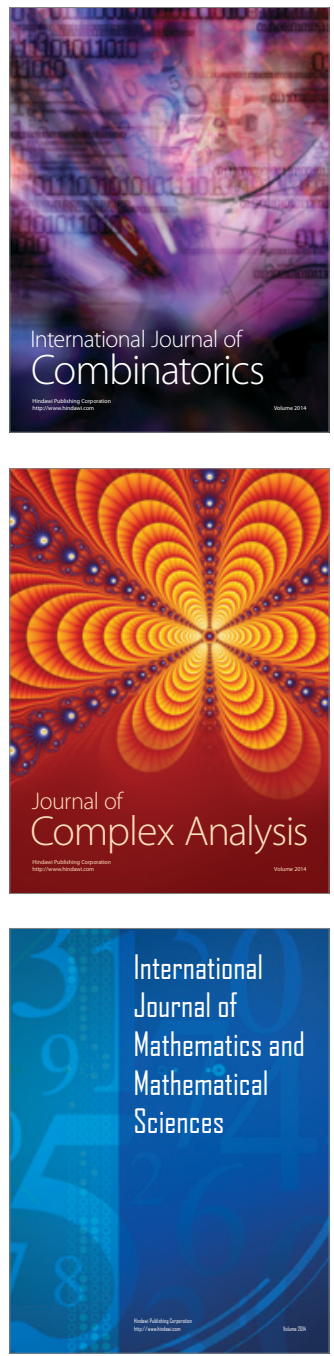
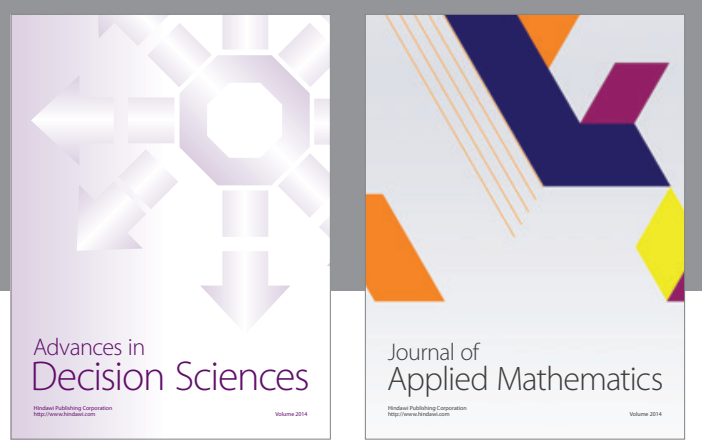

Algebra

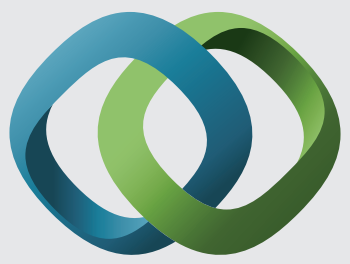

\section{Hindawi}

Submit your manuscripts at

https://www.hindawi.com
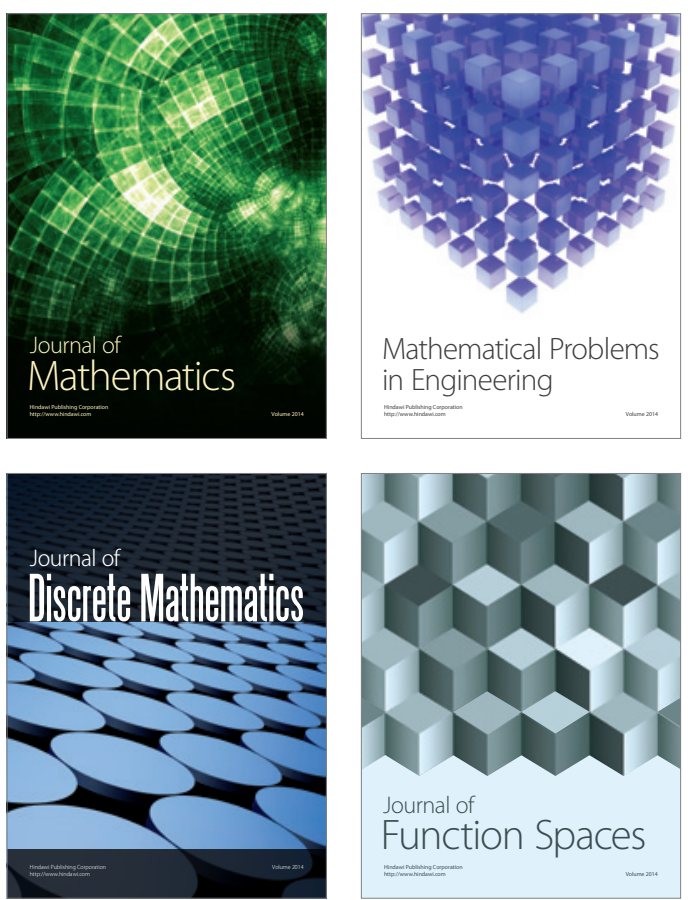

Mathematical Problems in Engineering
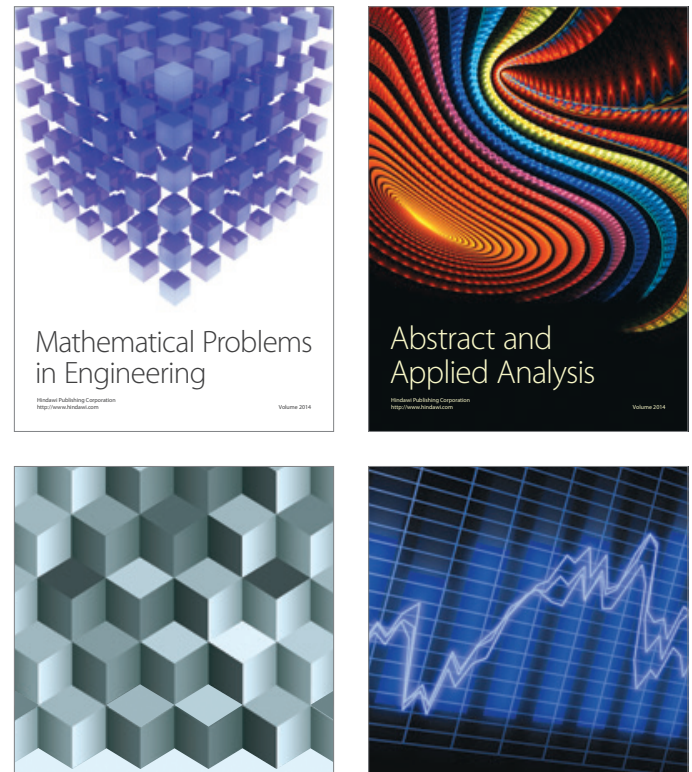

Journal of

Function Spaces

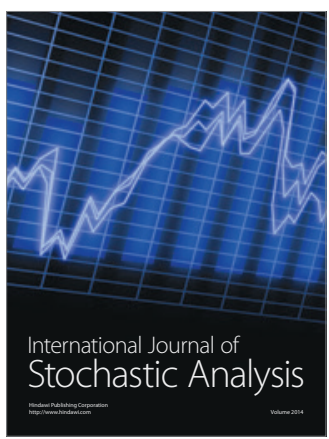

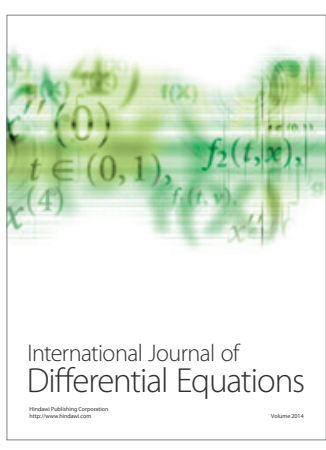
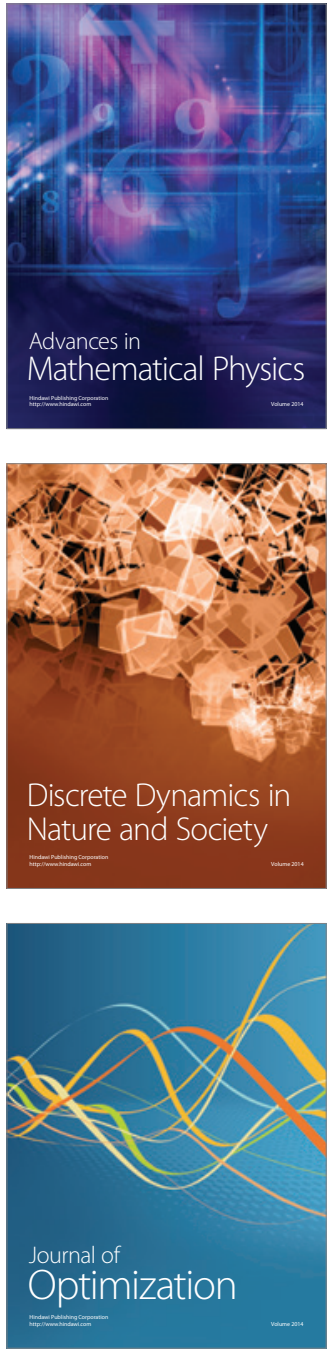